\title{
BMJ Open \\ Cross-cultural adaptation, evaluation and validation of the Spouse Response Inventory: a study protocol
}

\author{
Ulrike Kaiser, ${ }^{1}$ Dorit Steinmetz, ${ }^{1}$ Rüdiger Scharnagel, ${ }^{1}$ Mark P Jensen, ${ }^{2}$ \\ Friedrich Balck, ${ }^{3}$ Rainer Sabatowski ${ }^{1}$
}

To cite: Kaiser U, Steinmetz D, Scharnagel R, et al. Cross-cultural adaptation, evaluation and validation of the Spouse Response Inventory: a study protocol. BMJ Open 2014;4: e005119. doi:10.1136/ bmjopen-2014-005119

- Prepublication history for this paper is available online. To view these files please visit the journal online (http://dx.doi.org/10.1136/ bmjopen-2014-005119).

Received 24 February 2014 Revised 18 July 2014 Accepted 18 August 2014

\section{(a) CrossMark}

${ }^{1}$ Comprehensive Pain Center, University Hospital of Dresden, Dresden, Germany ${ }^{2}$ Department of Rehabilitation Medicine, Harborview Medical Center, Seattle, Washington, USA

${ }^{3}$ Clinic of Medical Psychology and Sociology, University

Hospital of Dresden,

Dresden, Germany

Correspondence to Dr Ulrike Kaiser;

ulrike.kaiser.usc-tkı@ uniklinikum-dresden.de

\section{ABSTRACT}

Introduction: Since the response of spouses has been proven to be an important reinforcement of pain behaviour and disability it has been addressed in research and therapy. Fordyce suggested pain behaviour and well behaviour be considered in explaining suffering in chronic pain patients. Among existing instruments concerning spouse's responses the aspect of well behaviour has not been examined so far. The SRI (Spouse Response Inventory) tries to consider pain behaviour and well behaviour and appears to be acceptable because of its brevity and close proximity to daily language. The aim of the study is the translation into German, followed by evaluation and validation, of the SRI on a German sample of patients with chronic pain.

Methods and analyses: The study is

comprehensively designed: initially, the focus will lie on the translation of the instrument following the guidelines for cross-cultural translation and adaptation and evaluation of the German version according to the source study. Subsequently, a validation referring to predictive, incremental and construct validation will be conducted using instruments based on similar or close but different constructs. Evaluation of the resulting SRI$\mathrm{G}$ (SRI-German) will be conducted on a sample of at least 30 patients with chronic pain attending a comprehensive pain centre. For validation at least 120 patients with chronic headache, back pain, cancer related pain and somatoform pain disorder shall be included, for a total of 480 patients. Separate analyses according to specific pain diagnoses will be performed to ensure psychometric property, interpretability and control of diagnosis of specific limitations. Analyses will include comprehensive investigation of psychometric property of the scale by hierarchical regression analyses, correlation analyses, multivariate analysis of variance and exploratory factor analyses (SPSS).

Ethics: The study protocol was approved by the Ethics Committee of the University of Dresden (EK 335 122008) based on the Helsinki declaration.

\section{INTRODUCTION}

The Spouse Response Inventory (SRI) follows the operant behavioural model of chronic pain first introduced by Fordyce. ${ }^{1}$ Fordyce suggested that pain behaviour (defined as all forms of behaviour indicating that a person is in pain, such as grimacing, groaning, limping, guarding, etc), as a strongly disabling behaviour, can come under control of reinforcing influences of the environment. Specifically, the model proposes that behaviours followed by reinforcers will increase in frequency, while those that are ignored or followed by punishers will decrease in frequency. He emphasised the role of significant others (such as spouses) in this process. ${ }^{2}$ Fordyce also defined well behaviour as a complementary form of behaviour that is not compatible with the role of an invalid person. He assumed that strengthening well behaviour would reduce pain related to sickness behaviour and reduce disability and suffering as well. Research that has examined the associations between spouse solicitous responses (generally assumed to be reinforcing) and patient pain behaviour has generally supported the operant model. ${ }^{3-9}$ However, although research found convincing evidence of the operant model with respect to the hypothesised positive association between spouse solicitous responses and measures of patients' pain and dysfunction, there is limited information regarding associations between environmental responses to patient well behaviours (eg, exercise) and measures of patient functioning.

Such operant mechanisms have been incorporated into multidisciplinary pain programmes and have influenced many of the established psychological approaches. ${ }^{10}$ Unfortunately, there have not been many instruments to evaluate such programmes according to these variables and, until now, the only existing ones are those that consider pain behaviour.

To overcome this constriction, Schwartz et $a l^{11}$ developed the SRI including one scale of spouses' responses to pain behaviour and one scale of spouses' responses to 
well behaviour following the definition put forth by Fordyce. ${ }^{1}$ Each of the scales is composed of a negative response ('negative' to pain and also well behaviour) and a positive response ('solicitous' to pain behaviour; 'facilitative' for well behaviour). The evaluation included 104 patients with unspecified chronic pain attending a multidisciplinary pain centre. Internal consistency was sufficient, ranging from 0.73 to 0.84 for all subscales, testretest stability ranged from 0.73 to $0.84 .{ }^{11}$ Item-scale correlation was reported between 0.56 and 0.86 . Measures of negative responses (presumed to be punishing) to patient well behaviour and solicitous responses to patient pain behaviour made independent contributions to the prediction of patient disability. At present, preliminary evidence supports the predictive validity and reliability of the SRI scales.

Pence $e t a l^{12}$ provided additional evidence on the validity of the SRI scales in a sample of patients with chronic headache. Specifically, they found that the SRI measure of spouse negative responses to well behaviour were significantly associated with greater patient pain behaviour and pain intensity. Further, they reported SRI measures of spouse solicitous responses to patient pain behaviour were associated with higher levels of pain behaviour, depressive symptoms, pain intensity and pain interference. ${ }^{12}$ Although preliminary, but consistent with the operant model of chronic pain, the evidence to date suggests that environmental (spouse) responses to patient pain and well behaviour both may play a role in patient adjustment to chronic pain.

A broader investigation of the present results, considering cultural differences, especially in marital interactions, seems to be helpful to the understanding of those relations between chronicity and environmental with respect to marital influencing variables. Unfortunately, there is a lack of instruments to measure these variables in German speaking populations and also in considering both dimensions of patients' behaviours. The most frequently used measure at present to assess spouse responses is the German version of the MPI ${ }^{13}$ which only assesses spouse responses to pain behaviour. Although a questionnaire developed by Leidig ${ }^{14}$ assesses perceived spouse response to pain and well behaviours, it is limited because it has never been used after publication, either in a German or any other sample and has not been further investigated for its psychometric properties. Similarly, a measure developed by Kröner-Herwig ${ }^{15}$ assesses aspects of spouses' responses to pain behaviour but again misses well behaviour. This measure, however, is limited by the absence of validation and evaluation investigations. An international discussion of the results has not yet been possible, but seems to be important in revealing cultural and regional differences in interactions and their impact on sickness or even chronic pain.

From the present point of view, the acknowledgment of the impact of interactions in significant relationships on chronicity seems to be necessary to understand processes of rehabilitation and sickness. The aim of the study is, therefore, the translation, evaluation and further validation of an existing instrument (SRI), along with the investigation of its cross-cultural application and psychometric properties.

\section{METHODS}

The design of the study is comprehensive and follows a multimethodical approach. Three parts will be conducted, consisting of translation and crosscultural adaptation of the SRI into German, a subsequent evaluation in a pilot trial and a validation considering aspects of construct validity, incremental validity, predictive validity, aspects of reliability and structure of the instrument.

\section{Part 1: Translation and cross-cultural adaptation Procedure}

The planned procedures for translating the SRI will be based on the Guidelines for Translation and Cross-cultural Adaptation by Beaton et $a l^{16}$ guidelines to evaluate and translate health status questionnaires according to Bullinger $e t a l^{17}$ and Guillemin $e t a l^{18}$ and will be performed in four steps:

1. Translation. Two independent persons will translate the SRI instructions and items from English into German at the same time. One will be aware of the study's purpose and goals, and the other will not, as recommended by Beaton et al. ${ }^{16}$

2. Synthesis. Both translators will discuss the differences between their translations to resolve any differences until they develop a consensus about the German wording of each item.

3. Back translation. Two back translations of two native speakers into English are generally recommended. However, in this case, it is assumed that only one back translation will be needed, because the SRI instructions and items are very straightforward.

4. Expert Committee Review. All study participants will then discuss any discrepancies found between the original SRI instructions and items and the backtranslated version.

Following the development of the first version of the SRI-G (SRI-German), a final test to evaluate the readability and grammar ${ }^{16}$ by a single testing is planned. Two methods are recommended to determine if patients (1) answer in a right form and also (2) understand the questions. ${ }^{16}$ The first method is to perform cognitive testing of the items (asking respondents after they complete each question if they understand what the question is assessing) by target sample. The second method is to ask bilingual individuals to complete the original as well as translated versions, and then perform cognitive testing asking them to provide their opinion regarding the similarity and readability of each version. Given the limited number of bilingual patients in the provided setting, the first method is chosen. The complete translation procedure will be fully documented to make it transparent for future discussion. 
Sample

Thirty to 40 patients shall be recruited for this part.

\section{Part 2: Psychometric evaluation of the SRI-G}

\section{Procedure}

The SRI-G will be administered to patients with chronic back pain who attend a multidisciplinary pain management programme. During an individual appointment the physician will inform the patient about the study. The patient will be invited to participate and those who wish to do so will be asked to read and sign a consent form. Patients who decline participation will be asked if they are willing to provide the basic demographic and pain history information in order to allow for comparisons between those who choose to participate and those who do not.

During the appointment the physician will document pain duration, age, gender, educational status and medical treatment history of participants. The physician will also complete the questions concerning pain behaviour, pain intensity and pain related disability. The participating patient will be administered a battery of questionnaires (spouse responses, depressive symptoms and disability) to complete after his or her appointment in the waiting area and be asked to return the completed forms to the study nurses. Both forms, the documentary form filled in by the investigators and the questionnaire, are provided with a special, individual code. There are no personal data on either form.

After 10 days, the retest form of the SRI-G will be mailed to the patient and he or she will be asked to return the instrument within 4 days. If the completed questionnaire has not arrived within 7 days, the patient will be reminded by letter once.

\section{Instruments}

Schwartz $e t a l^{11}$ evaluated the validity of the SRI scales by examining their associations with the following criterion variables:

- Disability (measured by the Roland and Morris disability questionnaire ${ }^{19}$ )

- Pain intensity (measured by numerical rating scale (NRS), 0-10)

- Pain behaviour (measured by the Pain Behaviour Checklist ${ }^{20}$ )

- Depression (measured by the Beck Depression Inventory $^{21}$ )

\section{Disability}

The Roland and Morris disability questionnaire (RMD) is available in the German language in two versions. ${ }^{22} 23$ The version of Wiesinger et al has been chosen since both versions have very similar results in testing the quality of the final version but Wiesinger et al has a more pleasant language usage. The PDI (Pain Disability Index ${ }^{24} 25$ ) is planned to be administered to extend and validate the findings by using another validated measure of disability.

\section{Pain intensity}

To measure pain intensity, participants will be asked to rate their average pain intensity over the last week on a 0-10 numeric rating scale ( 0 for no pain, 10 for worst pain imaginable). ${ }^{26} 27$

\section{Pain behaviour}

A German self-report pain behaviour measure, similar to the measure used in the original SRI scale development study (ie, the PBCL Pain Behaviour Check List ${ }^{20}$ ) is not available. The TBS (Tübinger Bogen zum Schmerzverhalten ${ }^{12}$ ) is perhaps the German measure that is most similar to the PBCL, although it will be completed by an observer and not by the patient. The physician will complete the TBS during the first appointment, after the patient has given his/her consent to participate.

\section{Depressive symptoms}

Depressive symptoms will be assessed by the German version of the BDI (Beck Depression Inventory), a frequently used and well-validated scale. ${ }^{21}$

\section{Sample characteristics}

Participants will be asked to provide basic demographic and pain history information (educational status, age, gender, medical treatment history and pain duration). Completers will be compared with those who declined to determine if any systematic differences in basic demographic and pain history have occurred.

\section{Sample}

According to the calculation of sample sizes for regression analysis ${ }^{28} 116$ individuals will be recruited (including a calculated drop-out rate of 20\%). Recruitment will be conducted by the Comprehensive Pain Centre at the University Hospital of Dresden.

\section{Inclusion criteria}

Outpatients (aged between 18 and 70 years) attending the comprehensive pain centre and suffering from chronic pain with a duration of at least 6 months will be asked to participate. Participants have to live in a relationship and fluent German language will be required. Written confirmed consent will be necessary.

\section{Exclusion criteria}

Patients who are not involved in a relationship or who do not provide informed and written consent will be excluded, as will patients with insufficient German language skills.

\section{Analyses}

Schwartz et $a l^{11}$ presented means and SDs, internal consistency (Cronbach $\alpha$ ), test-retest stability coefficients, item-scale intercorrelation and interscale correlations, and finally performed a factor analysis (scree test) to evaluate the factor structure of the SRI items. This study will follow to ensure comparability of results. Data will 
be entered into a data file and analysed using a SPSS statistical program (V.21.0).

To ensure factor structure of the SRI-G, an explanatory factor analyses will be conducted (principal component analyses, VARIMAX rotation) and the results will be compared to the source study.

To examine the stability of the resulting SRI-G scales, test-retest stability coefficients will be computed, with further Cronbach's $\alpha$. Predictive validity will be estimated through a series of regression analyses (hierarchical model) in predicting depressive symptoms (BDI), pain behaviour (TBS), pain intensity (NRS) and disability (RMD).

\section{Part 3: validation of the SRI}

Schwartz et $a l^{11}$ validated the instrument on the basis of the predictive value of the SRI according to the literature and proved its predictive validity sufficiently. However, it appears to be necessary to have more information on construct validity (how much does the instrument measure what it is supposed to measure) and its subtype incremental validity (what does the instrument add to previous knowledge).

\section{Procedure}

All patients who attend the comprehensive pain centre will be asked to participate. During an individual appointment the physician will inform the patient about the content and aim of the study. The patient will be asked to give his written consent. Patients who refuse to participate will be listed with age, gender, educational status, actual medical treatment and pain duration to control selection bias.

During the appointment the physician documents the pain duration, age, gender, educational status, pain behaviour, pain intensity and actual medical treatment. After the appointment, the participating patient receives a questionnaire to complete in the waiting area and is asked to return it to the study nurses after completion. To fill in the questionnaires will require approximately between 35 and $45 \mathrm{~min}$.

Both forms, the documentary form and the questionnaire, are provided with a special, individual code. There are no personal data on either form. The data will be electronically saved.

\section{Instruments}

\section{Predictive validity}

To compare this study with part 2 and the source study of Schwartz et al, the same measure (pain behaviour (TBS), depressive Symptoms (BDI), pain intensity (NRS) and disability (PDI) ) as described above will be used. The Roland and Morris Questionnaire will be replaced by the PDI because the PDI refers to unspecified chronic pain.

\section{Incremental validity}

There are many instruments that focus on interactions between spouses. Most of them are not specific for pain patients and their partners, but since all interactions are human, whether with or without pain, the assumption of similarities between an interaction between healthy spouses and an interaction between healthy and sick spouses seems to be comprehensible. A new instrument shall prove that it is able to reveal more specific information about the subject than previous ones to justify its use.

To ensure this part of validation three questionnaires from social science with different concepts were chosen: the concept of social support (F-Sozu), ${ }^{29}$ the concept of marital satisfaction ${ }^{30} 31$ and the concept of family emotional involvement and criticism. ${ }^{32-34}$ All these concepts have proven to relate to depression, pain intensity, pain behaviour and disability. ${ }^{8} 735-40$

\section{Construct validity}

Under this perspective the instrument shall be similar to other instruments claiming the measurement of the same subject. In the case of the SRI we include two similar instruments in German (see above): the spouse's response questionnaire by Leidig ${ }^{14}$ and the German Multidimensional Pain Inventory. ${ }^{41}$ Similarities between these instruments and the SRI shall ensure its construct validity; however, there remains the dilemma that an overlap of the instruments does not justify a new instrument.

The complete measurement contains the following instruments:

A. SRI: (ref. ${ }^{11}$, in translation)

B. MPI-D: West Haven-Yale Multidimensional Pain Inventory (subscale perceived spouse response to pain behaviour) ${ }^{13} 4142$

C. FPS: Fragebogen zu Partnerschaft und Schmerz; Questionnaire of spouse response to pain and well behaviour $^{14}$

D. FEIWK: Fragebogen zur familiären emotionalen Involviertheit und wahrgenommenen Kritik; German version of the Questionnaire of family emotional involvement and perceived criticism ${ }^{32-34}$

E. F-SozU: Fragebogen zur sozialen Unterstützung; Questionnaire of social support ${ }^{29}$

F. FBZ: Fragebogen zur Zweierbeziehung; German version of the Dyadic Adjustment Scale ${ }^{31} 43$

G. BDI: Beck Depression Inventory; German version ${ }^{21} 44$

H. PDI: Pain Disability Index; German version ${ }^{24} 45$

\section{Sample}

Based on the calculation of optimal sample $\operatorname{sizes}^{28}$ for regression analyses, 116 patients (inclusive of drop-out of $20 \%$ ) shall be recruited into one group.

\section{Inclusion criteria}

Patients of the multidisciplinary pain management centre will be contacted when they suffer from:

A. chronic back pain,

B. chronic tension headache or migraine

C. somatoform pain disorder

D. cancer pain in a palliative care setting in our Cancer Centre and when patients present a Karnofsky-index of at least $80 \%$ 
Patients have to live in a relationship, be between 17 and 70 years of age, and speak German fluently. Only patients who provide informed and written consent will participate.

\section{Exclusion criteria}

Patients who have not given their informed and written consent will be excluded. Also, patients who have more than one pain syndrome, who are not involved in a relationship, or who do not have adequate German language skills and who have participated in earlier steps of the study will be excluded from participation.

\section{Analyses}

All analyses will be performed for the whole sample of included patients and further for pain diagnoses separately. Since the population of chronic pain patients is very heterogeneous it seems to be helpful to distinguish possible differences in results according to pain diagnoses.

Construct validity will be analysed by comparison between the MPI-D subscale 'spouse responses' and the FPS referring to the SRI. Methodological problems remain in the structure of these instruments: the MPI contains only one perspective (spouse response to pain behaviour), the FPS contains two perspectives (spouse response to pain and to well behaviour) but with slightly different conceptual explanations. The disadvantage of the FPS is a lack of empirical experience because it has not been investigated sufficiently.

The final scores of the different subscales will be compared by correlation analyses.

Predictive validity will be analysed according to the source study. ${ }^{11}$ The process is the same as described above for part 2.

According to the previous literature, the SRI seems to support the relationship between spouse response and depression, disability, pain intensity and pain behaviour. ${ }^{11}$ These results shall be expected. Additionally, the FPS and the MPI-D will be included into the same analysis to investigate their amount of predictive validity on these parameters. For the investigation of the predictive validity a regression analysis (hierarchical model) is scheduled.

Incremental validity of the concerning instruments (FEIWK, FBZ, F-Sozu, SRI, MPI-D and FPS) will be determined by a correlation analysis and stepwise (forward) regression analyses.

All analyses will be done by SPSS V.21.0.

\section{Timeframe of the study}

The translation process was completed in June 2008 and the testing of the final version was completed in August 2008. Data collection for evaluation was completed by June 2010 and exploratory data analysis by December 2010.

The validation study including headache patients started in February 2009 and finished at the end of
2013. The study with patients in palliative care started in April 2009, completion is planned to be in summer of 2014. The studies with back pain and patients with somatoform pain disorder started between spring and summer of 2010 and will be finished by summer of 2014 at the latest. Data analyses will follow after completing recruitment.

\section{Description of risks}

There will be no risks for included patients.

\section{Ethical and legal aspects \\ Ethical principles}

The study is being conducted in accordance with medical professional codex and the Helsinki Declaration as of 1996 as well as the German Federal Data Security Law. Study participation by patients is voluntary and can be cancelled at any time without provision of reasons and without negative consequences to their future medical care.

\section{Patient informed consent}

Previous to study participation patients receive written and oral information about the content and extent of the study. They sign the informed consent form to accept.

\section{Legal principles}

\section{Data security and disclosure of original instruments}

After the questionnaires and documents, which are provided with a special code, have been completed, they will be stripped of personal data (names, date of birth, etc). Personal data will be eliminated. All electronical data of the complete, coded documents will be saved on a protected server. Only members of the internal study team can access the respective files. The paper form of the documents will be stored in archives closed for external persons.

Acknowledgements The authors would like to thank Mrs Susan Gubitz (Cologne) for her support in the translation process.

Contributors UK, DS, FB, RSa and MPJ were involved in the general study design and the writing of the manuscript. RSc and DS contributed to substudies on different pain aetiologies. UK and RSa were involved in the translation process.

\section{Competing interests None.}

Patient consent Obtained.

Ethics approval The study protocol was approved by the Ethics Committee of the University of Dresden. (Translation, Cross-Cultural Adaptation and Evaluation number: Validation study: EK 335 122008).

Provenance and peer review Not commissioned; externally peer reviewed.

Data sharing statement No additional data are available.

Open Access This is an Open Access article distributed in accordance with the Creative Commons Attribution Non Commercial (CC BY-NC 4.0) license, which permits others to distribute, remix, adapt, build upon this work noncommercially, and license their derivative works on different terms, provided the original work is properly cited and the use is non-commercial. See: http:// creativecommons.org/licenses/by-nc/4.0/ 


\section{REFERENCES}

1. Fordyce WE. Behavioral methods for chronic pain and illness. St. Louis: Mosby, 1976

2. Fordyce WE. Effects of marital interaction on chronic pain and disability: examining the down side of social support. Comment Rehab Psychol 1993;38:211-12.

3. Block AR, Kremer E, Gaylor M. Behavioral treatment of chronic pain: the spouse as a discriminant cue for pain behavior. Pain 1980;9:243-52.

4. Flor $\mathrm{H}$, Kerns RD, Turk DC. The role of spouse reinforcement, perceived pain activity levels in chronic pain patients. J Psychosom Res 1987;31:251-9.

5. Lousberg R, Schmidt AJ, Groenman NH. The relationship between spouse solicitousness and pain behavior: searching for more experimental evidence. Pain 1992;51:75-9.

6. Romano JM, Jensen MP, Turner JA, et al. Chronic pain patient-partner interactions: further support for a behavioral model of chronic pain. Behav Ther 2000;31:415-40.

7. Romano JM, Turner JA, Friedman LS, et al. Sequential analysis of chronic pain behaviors and spouse responses. J Consult Clin Psychol 1992;60:777-82.

8. Cano A, Gillis M, Heinz W, et al. Marital functioning, chronic pain, and psychological distress. Pain 2004;107:99-106.

9. Romano JM, Turner JA, Jensen MP, et al. Chronic pain patient-spouse behavioral interactions predict patient disability. Pain 1995;63:353-60.

10. Flor H, Turk DC. Chronic pain. An integrated biobehavioral approach. Seattle: IASP Press, 2011.

11. Schwartz L, Jensen MP, Romano LM. The development and psychometric evaluation of an instrument to assess spouse response to pain and well behavior in patients with chronic pain: the spouse response inventory. J Pain 2005;6:243-52.

12. Pence LB, Thorn BE, Jensen MP, et al. Examination of perceived spouse responses to patient well and pain behavior in patients with headache. Clin J Pain 2008;24:654-61.

13. Flor H. Psychobiologie des Schmerzes [Psychobiology of pain]. Göttingen: Verlag Hans Huber, 1991.

14. Leidig U. Chronischer Schmerz und Partnerschaft [Chronic pain and partnership]. Aachen: Shaker Verlag, 1996.

15. Kröner-Herwig B. Rückenschmerz [Back pain]. Göttingen: Hogrefe, 2000.

16. Beaton DE, Bombardier C, Guillemin F, et al. Guidelines for the process of cross-cultural adaptation of self-report measures. Spine 2000;25:3186-91.

17. Bullinger M, Alonso J, Apolone $\mathrm{G}$, et al. Translating health status questionnaires and evaluating their qualities: the IQOLA project approach. J Clin Epidemiol 1998;51:913-23.

18. Guillemin F, Bombardier C, Beaton DE. Crosscultural adaptation of health-related quality of life measures: literature review and proposed guidelines. J Clin Epidemiol 1993;46:1417-32.

19. Roland M, Morris R. Development of a reliable and sensitive measure of disability in low back pain. Spine 1983;8:141-4.

20. Kerns RD, Haythornthwaite J, Rosenberg R, et al. The pain behavio checklist (PBCL): factor structure and psychmetric properties. J Behav Med 1991;14:155-67.

21. Beck AT, Ward $\mathrm{CH}$, Mendelson $\mathrm{M}$, et al. An inventory for measuring depression. Arch Gen Psychiatry 1961;4:461-571.

22. Wiesinger GF, Nuhr M, Quittan M, et al. Cross-cultural adaptation of the Roland-Morris questionnaire for German-speaking patients with low back pain. Spine 1999;24:1099-108.

23. Exner V, Keel P. Erfassung der Behinderung bei Patienten mit chronischen Rückenschmerzen. Validierung einer deutschen Version des "Roland \& Morris disability questionnaire" sowie verschiedener Ratingskalen [Measuring disability of patients with low-back pain-validation of a German version of the Roland \& Morris disability questionnaire]. Schmerz 2000;14:392-400.

24. Dillmann $U$, Nilges $P$, Saile $H$, et al. Behinderungseinschätzung bei chronischen Schmerzpatienten [Evaluation of disability in chronic pain patients]. Der Schmerz 1994;8:100-10.
25. Grönblad M, Hupli M, Wennerstrand $P$, et al. Intercorrelation and test-retest reliability of the pain disability index and the Oswestry disability questionnaire and their correlation with pain intensity in low back pain patients. Clin J Pain 1993;9:189-95.

26. Jensen MP, Karoly $P$, Braver $S$. The measurement of clinical pain intensity: a comparison of six methods. Pain 1986;27: 117-26.

27. Kremer E, Atkinson JH, Ignelzi RJ. Measurement of pain: patient preference does not confound measurement. Pain 1981;10:241-8.

28. Bortz J. Statistik [Statistics]. 4th edn. Heidelberg: Springer, 1999

29. Fydrich T, Sommer G, Brähler E. Fragebogen zur sozialen Unterstützung F-SozU [Questionnaire to assess social support F-SozU]. Göttingen: Hogrefe, 2007.

30. Hank G, Hahlweg K, Klann N. Diagnostische Verfahren für Berater. Materialien zur Diagnostik und Therapie in Ehe-, Familien- und Lebensberatung [Diagnostic methods in marital, familial and life counselling]. Weinheim: Beltz, 1990.

31. Spanier GB. Measuring dyadic adjustment: new scales for assessing the quality of marriage and similar dyads. J Marriage Fam 1976;12:15-28.

32. Kronmüller K-T, Krummheuer C, Hartmann M, et al. Der Fragebogen zur familiären emotionalen Involviertheit und wahrgenommenen Kritik (FEIWK). Ein Verfahren zur Erfassung von Expressed Emotion [The questionnaire of familial emotional involvement and perceived criticism (FEIWK). An approach to assess expressed emotion]. Psychother Psychosom Med Psychol 2000;51:337-83.

33. Shields CG, Franks P, Harp JJ, et al. Development of the Family Emotional Involvement and Criticism Scale (FEICS): a self-report scale to measure expressed emotion. J Marital Fam Ther 1992;18:395-407.

34. Shields CG, Franks P, Harp JJ, et al. Family Emotional Involvement and Criticism Scale (FEICS): II. Reliability and validity studies. Fam Syst Med 1994;12:361-77.

35. Kerns RD, Haythornthwaite J, Southwick S, et al. The role of marital interaction in chronic pain and depressive symptom severity. J Psychosom Res 1990;24:401-8.

36. Cano A, Weisberg JN, Gallagher RM. Marital satisfaction and pain severity mediate the association between negative spouse responses to pain and depressive symptoms in a chronic pain patient sample. Pain Med 2000;1:35-43.

37. Cano A, Johansen AB, Franz A. Multilevel analysis of couple congruence on pain, interference, and disability. Pain 2005;118:369-79.

38. Geisser ME, Cano A, Leonard M. Factors associated with marita satisfaction and mood among spouses of persons with chronic back pain. J Pain 2005;6:518-25.

39. Fydrich $\mathrm{T}$, Bastian $\mathrm{H}$, Flor $\mathrm{H}$. Die Rolle der Familie bei chronischen Schmerzen [The impact of family on chronic pain]. In: Basler H-D, Franz C, Kröner-Herwig B, Rehfisch HP, Seemann H, eds. Psychologische Schmerztherapie. Berlin: Springer, 1998: 213-23.

40. Jamison RN, Virts KL. The influence of family support on chronic pain. Behav Res Ther 1990;28:283-7.

41. Flor H, Rudy TE, Birbaumer N, et al. Zur Anwendbarkeit des West Haven-Yale Multidimensional Pain Inventory im deutschen Sprachraum [Application of the West Haven-Yale Multidimensiona Pain Inventory in German]. Der Schmerz 1990;4:82-7.

42. Kerns RD, Turk DC, Rudy Th E. The West Haven-Yale Multidimensional Pain Inventory (WHYMPI). Pain 1985;23:345-56.

43. Klann N, Hahlweg K, Heinrichs N. Diagnostische Verfahren für die Beratung [Diagnostic methods in counselling]. 2nd edn. Göttingen: Hogrefe, 2003.

44. Hautzinger M, Bailer M, Worall $\mathrm{H}$, et al. Das Beck-DepressionsInventar (BDI) [The Beck Depression Inventory]. Bern: Huber Verlag, 1992.

45. Tait RC, Chibnall JT, Krause S. The pain disability index: psychometric properties. Pain 1990;40:171-82. 